The authors make it clear that late onset periventricular leucomalacia is three times more common than early onset cases. It is worth pointing out that this was not the situation that was apparent to perinatal pathologists in the days before ultrasonography existed. Only recently have significant numbers of small babies been kept alive long enough for periventricular cavitation to become manifest either by ultrasound or by direct observation postmortem. For these babies postnatal management is more likely to be relevant to their handicap than antepartum or intrapartum events. In historical terms antepartum and intrapartum brain damage has always afflicted the human race and is probably diminishing in western society. Late onset cerebral infarction in very prematurely born infants is a recent, iatrogenic problem whose increasing frequency lends greater urgency to its solution.

A J BARSON

$$
\begin{array}{r}
\text { Department of Pathology, } \\
\text { St Mary's Hospital, } \\
\text { Whitworth Park, } \\
\text { Manchester M13 ofH }
\end{array}
$$

1 Sinha SK, D'Souza SW, Rivlin E, Chiswick ML. Ischaemic brain lesions diagnosed at birth in preterm infants: clinical events and develop1017-20. but as the progression of initial echogenicity to cyst formation takes sometime between seven to 10 days, it seemed quite reasonable to deduce that these changes might have started before or during birth.

The ability to make serial observations of the brain non-invasively by ultrasound has led to a better understanding of the pathogenesis and natural history of ischaemic brain lesions. We agree that these lesions tend to occur mostly in relation to hypoxic-ischaemic injury either at birth or during the neonatal period but similar insults can occur antenatally. This notion is supported by a number of intrauterine Doppler investigations, and there is evidence both from human and animal studie showing damage to fetal periventricular white matter in response to various forms of hypoxicischaemic insults. The fact that in a third of the babies in our study, who later developed disabilities, the brain damage may have already taken place by the time of birth has important implications and should not be ignored. Indeed, at all gestational ages, perinatal problems represent a continuum including prenatal, intrapartum, and neonatal events.

Neonatal infections with coagulase negative staphylococci

SIR,-In their recent leading article Millar et al state that resistance of coagulase negative staphylococci to penicillin, aminoglycosides, and other antibiotics is common.

This is not our experience at Liverpoo Maternity Hospital where coagulase negative staphylococci are responsible for most proved neonatal infections. ${ }^{2}$ When any infection is suspected, our practice is to treat initially with ampicillin and gentamicin until the results of cultures are available after which treatment may be adjusted according to antibiotic sensitivities. Occasionally the infant has coagulase negative staphylococci infection and has clinically improved despite in vitro resistance to ampicillin and gentamicin. If this is the case these antibiotics are often continued with good effect confirming a discrepancy between in vivo and in vitro sensitivities.

Since 1986 we have used amoxycillin/ clavulanic acid (Augmentin, Beecham) for coagulase negative staphylococci infections caused by penicillin, methicillin, and gentamicin resistant organisms when the initial ampicillin and gentamicin combination has failed. In a series of 70 epidsodes of coagulase negative staphylococci septicaemia occurring between January and July 1987 only one strain was found to be resistant to amoxycillin clavulanic acid. The majority of strains were $\beta$-lactamase producers.

Coagulase negative staphylococci infection cannot usually be differentiated clinically from other neonatal infection; using vancomycin to treat suspected coagulase negative staphylococci infection as suggested by Millar et al means using it in virtually all episodes of suspected neonatal infection. From our clinical and laboratory experience we do not agree that vancomycin, which is ototoxic nephrotoxic, has to be given by infusion and is expensive, should be the drug of first choice for the treatment of suspected coagulase negative staphylocci infection in the neonate.

N J SHAW

V DAMJANOVIC A M WEINDLING

Regional Neonatal Intensive Care Unit, Department of Child Health, Department of Child Health, Oxford Street

Liverpool L7 7NB

1 Millar MR, Todd N, Mackay P. Neonatal infecve staphylococci. Arch Dis Child 1990;65:1015-6.

2 Hensey OJ, Hart CA, Cooke RWI. Serious infections in a neonatal care unit: a two year survey. f Hyg (Lond) 1985;95:289-97.

\section{Dr Millar comments}

Vancomycin is active in vivo and in vitro against 'methicillin resistant' coagulase negative staphylococci and side effects are well documented. Coagulase negative staphylococci infection is uncommon in the first week of life, in the absence of an intravascular catheter, and in the mature neonate, so it is certainly not necessary to use vancomycin for the treatment of all episodes of suspected neonatal infection. The use of amoxycillin clavulanic acid (Augmentin) for the treatment of neonatal infections as advocated by Shaw $e$ $a l$ is indeed unusual. Neither of the two components are active in vivo or in vitro against 'methicillin resistant' staphylococci when used as single agents. The implication of Shaw et al (although not stated) is that resistance of 'methicillin resistant' coagulase negative staphylococci to amoxycillin is determined by $\beta$-lactamase synthesis and that the altered affinity of penicillin binding proteins for $\beta$-lactamase antibiotics does not contribute to amoxycillin resistance. If the hypothesis that amoxycillin/clavulanic acid is effective against 'methicillin resistant' coagulase negative staphylococci in vivo or in vitro can be substantiated then those data should be published.

\section{Endotoxin induced cochlear damage}

SIR,-We read with interest the paper by Tarlow et al describing the cochlear damage in guinea pigs produced by endotoxin perfusion of the cochlea or injection into the cerebrospinal fluid. Two points are worth making.

Firstly, the concentration of endotoxin in the cerebrospinal fluid or the cochlea is not estimated, but may be several orders of magnitude greater than that in human meningitis. For example, concentrations in cerebrospinal fluid compatible with survival in meningococcal meningitis were $85-250 \mathrm{pg} / \mathrm{ml}$ in one study. ${ }^{2}$ It would be valuable to know whether cochlear toxicity in the guinea pig occurs at endotoxin concentrations in the cerebrospinal fluid comparable with those found during human meningitis.

Secondly, endotoxin mediation of cochlea damage might have important implications for children with Gram negative septicaemias as well as those with meningitis. We have seen a child who first complained of unilateral tinnitus and deafness 12 hours after presenting with meningococcal septicaemia. He had no clinical or laboratory evidence of meningitis Subsequent audiology confirmed complete left sided hearing loss. This damage could have been mediated by endotoxin circulating in plasma.

To test the hypothesis clinically that 